\title{
Outcome of Primary Ilizarov External Fixator in Gustilo Anderson Type II, IIIa and IIIb Tibial Fractures
}

\author{
Dr.Harendra Kumar ${ }^{1}$, Dr. Soumya Ghosh ${ }^{2}$, Dr. Pradip Kumar Ghosh ${ }^{3}$, \\ Dr. Sarita Sinha ${ }^{4}$, Dr. Arnab Sarkar ${ }^{5}$, Dr.Chiranjit De ${ }^{6}$, Dr.Apurba Gorain ${ }^{7}$ \\ ${ }^{2,3}$ (Orthopaedics,Bmch,India)Associate Professor \\ ${ }^{4}$ (Obstetrics And Gynaecology,Mamc,India)Seniour Resident \\ ${ }^{1,5,6,7}$ (Orthopaedics, Bmch,India)Juniour Resident.
}

\begin{abstract}
Open fractures are more common in the tibia than in any other bone, due to its location and tenuous soft tissue coverage. IEF is a step forward in the management of open tibial fractures in the settings where patients present late, have bone loss, and facilities for emergency nailing or bypass plating and assistance of plastic surgery are not available.. The outcome of treatment evaluated by both clinically \& radiologically using Johner and Wruh's criteria. Total 15 patients were included in the study satisfying the inclusion criteria. At the time of initial presentation, Gustillo-Anderson type IIIa \&IIIb was encountered in 13 patients(86.66\%) while type II was encountered in 2 patients (13.34\%). Union was achieved in all patients. The most common complication was superficial pin tract infection (20\%)that resolve with oral antibiotics, stiffness of knee $6.67 \%$ , stiffness of ankle $13.34 \%$ and $L L D<1 \mathrm{~cm}$ in $20 \%$ cases.Based on Johner\&Wruh's criteria, the final result was excellent in 2 patients(13.33\%), good in 10 patients(66.67\%), fair in 2 patients(13.33\%), and poor in 1 patient $6.67 \%$ in our study. The IEF is a minimally invasive method which permits effective wound management and early weight bearing.
\end{abstract}

Keyword: Ilizarov external fixator, Johner \&Wruch's criteria, Minimal invasive, Open fracture, Tibia.

\section{Introduction}

Open fractures are more common in the tibia than in any other bone, due to its location and tenuous soft tissue coverage ${ }^{1}$. Despite its frequency, the ideal management of open tibial fractures remains controversial. Modern day management of open injury has focused on early internal or external fixation with adequate soft tissue coverage when needed to enable early mobilization and restoration of optimum function ${ }^{2}$. Plate fixation and the conventional half-pin fixators are associated with high rates of non-union and the need for secondary procedures ${ }^{3}$. In the developed countries, primary debridement and intramedullary nailing or bypass plating is now increasingly becoming the preferred treatment of these fractures. However, in developing countries such as India, where the patients present late to the hospitals and adequate facilities (in terms of manpower and theatre facilities) are not always available, the situation is different. In our hospital, as in most centers in the less developed regions, open tibial fractures have been traditionally managed by external fixators. The high rate of failure associated with this management protocol made us to look to the Ilizarov external fixator (IEF) as an alternative. IEF is a step forward in the management of open tibial fractures in the settings where patients present late, have bone loss, and facilities for emergency nailing or bypass plating and assistance of plastic surgery are not available. The IEF is a minimally invasive method which permits effective wound management, early weight bearing and definitive treatment for open fractures ${ }^{4,5}$.

\section{Aim}

Assessment of results in terms of functional and radiological outcomes and complications of Ilizarov external fixator, so that proper management in the specific direction can be provided for better functional outcome and to minimise complication.

\section{Materials And Methods}

All patients attending Orthopaedics emergency/ OPD within the age group 18-60 years with open fractures of tibia in Burdwan Medical College and Hospital till sample size is obtained( 15 patients).Study period was jan 2015 to june 2016.patients with head trauma and neurovascular injury in same limb excluded in study. The outcome of treatment evaluated by both clinically \& radiologically using Johner and Wruh's criteria 

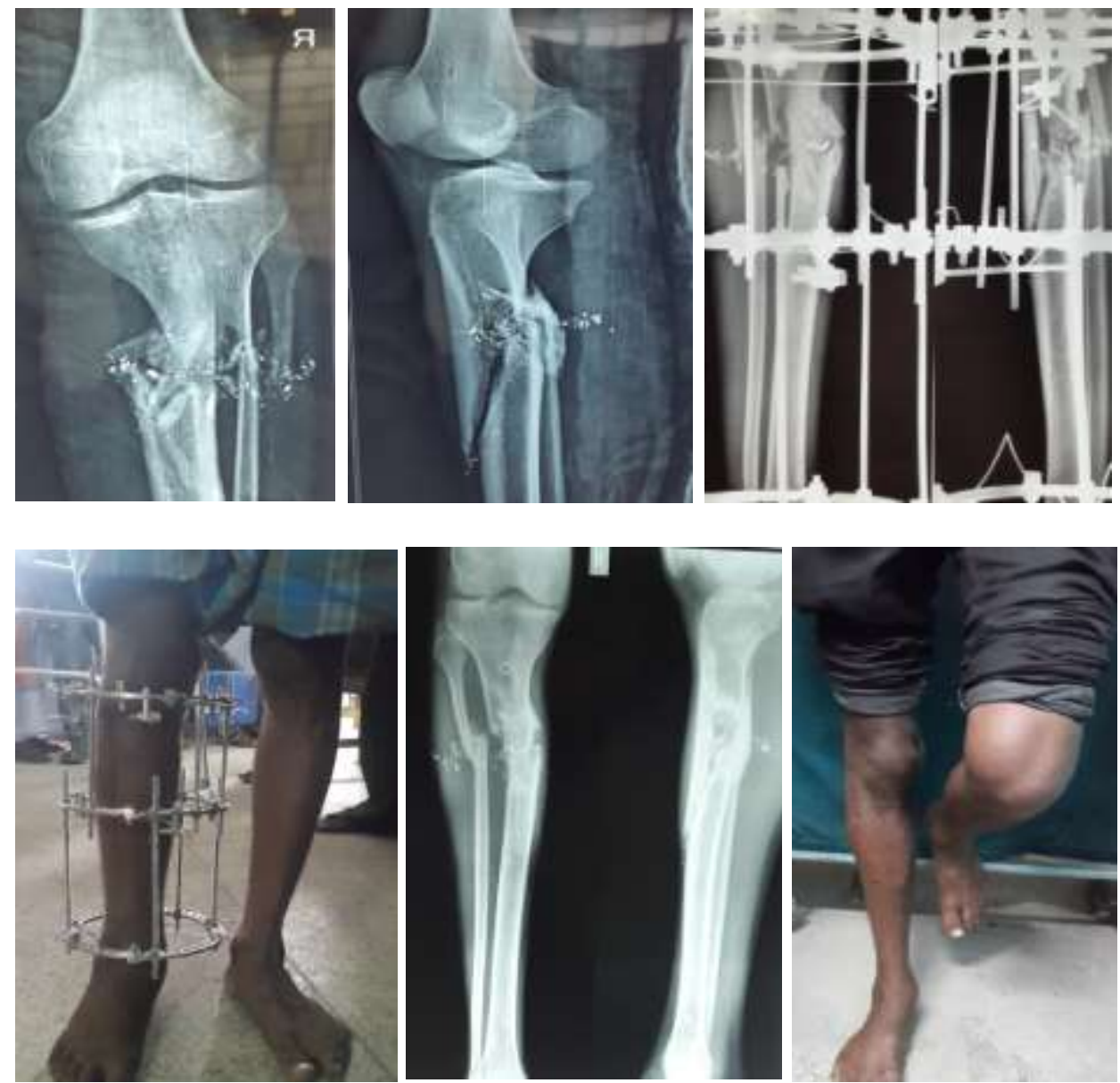

Figure

\section{Result}

During the study period, total 15 patients were included in the study satisfying the inclusion criteria.Age of the patients varied from 19 years to 56 years. Mean age is 36.54 years.Maximum incidence $(53.34 \%)$ was in the age group 31-40 years. Sex distribution of patients (13 male \& 2 female) showed male predominance( $86.66 \%)$.Rt. $\operatorname{Leg}(80 \%)$ was more commonaly involved than 1 t. $\operatorname{Leg}(20 \%)$. The study shows, RTA as the predominant cause. 13 patients had RTA $(86.66 \%), 1$ pt. had bomb blast injury $(6.67 \%)$ and 1 pt. had fall from height $(6.67 \%)$. At the time of initial presentation, Gustillo-Anderson type IIIa \&IIIb was encountered in 13 patients(86.66\%) while type II was encountered in 2 patients (13.34\%). According to AO classification of fractures, 5 patients had type A fractures(33.34\%), 9 patients had type B fractures(60\%) and 1 patient had type C fracture $(6.66 \%)$.

Mean value of ISI was 11.14 days .9 patients had been operated between 11-15 days (60\%), 5 patients had been operated between 6-10 days (33.34\%) and 1 patient had been operated between 16-20 days(6.66\%).The ilizarov fixator was kept for an average period of 186.86 days, the range being 121-238 days.The majority of patients (53.34\% ) had the fixator for 181-210 days. The mean duration of follow up was 12 months.Union was achieved in all patients. The most common complication was superficial pin tract infection (20\%)that resolve with oral antibiotics, stiffness of knee $6.67 \%$, stiffness of ankle $13.34 \%$ and LLD $<1 \mathrm{~cm}$ in $20 \%$ cases.Based on Johner\&Wruh's criteria, the final result was excellent in 2 patients(13.33\%), good in 10 patients(66.67\%), fair in 2 patients(13.33\%) , and poor in 1 patient $(6.67 \%$ in our study

\section{Conclusion}

This short term study deduced, Ilizarov external fixator is a versatile apparatus to provide primary definite fixation for open tibial fractures.Early weight bearing even in severly comminuted fractures is the key factor that separates it from other methods of fixation. It promote early functional recovery. Being a definitive method of fixation it excludes the need of second definitive fixation as in cases of conventional external fixation. 


\section{Aknowledgement}

Sincere thanks to patients and their relatives for patience and cooperation

\section{Limitation}

The sample size was small. In future it may be solved by increasing the number of cases and duration of follow up period.

\section{References}

[1]. Nicol SA. Closed and open management of tibial fractures. Clin Orthop Relat Res. 1974;105:144-153. doi: 10.1097/00003086197411000-00009

[2]. Gustilo RB, Markow RL, Templeman Current concept review: the management of open fractures. J Bone Joint Surg Am. 1990;72:299-303

[3]. Bilat C, Leutenegger A, Ruedi T. Osteosynthesis of 245 tibial fractures, early and late complications. Injury. 1994;25:349-358. doi: 10.1016/0020-1383(94)90125-2

[4]. Sidhartan S, Sujith A, Rathod AK, Pathak RH (2005) Management of high energy tibial fractures using the Ilizarov apparatus. Internnet J Orthop Surg 2(2).

[5]. Hosny G, Fadel M , Ilizarov external fixator for open fractures of the tibial shaft. Int Orthop,2003;23(5):303306.doi:10.1007/s00264-003-0476-3. 